\title{
Neue Maßnahmen gegen Gewaltvorkommnisse auf dem Fußballplatz im Amateurbereich.
}

\author{
Eine Forschungsskizze und erste Ergebnisse
}

Thaya Vester

Der 15. Mai 2012 war in negativer Hinsicht ein denkwürdiger Tag in der deutschen Fußballgeschichte. Beim Rückspiel der Relegation der ersten Fußball-Bundesliga zwischen den Vereinen Hertha BSC und Fortuna Düsseldorf kam es neben einem verfrübten Platzsturm von freudetrunkenen Düsseldorfer Fans zu Attacken gegen den Schiedsrichter Wolfgang Stark durch Berliner Spieler. Insbesondere ein Faustschlag von hinten im Kabinengang durch den Spieler Levan Kobiaschwili sorgte für großes Aufsehen, nicht zuletzt auch deshalb, da dieses Vergehen eine Rekordsperrstrafe in der Bundesliga nach sich zog. Schließlich handelte es sich bei diesem Sachverhalt um eine unglaubliche Entgleisung gegen einen Schiedsrichter im deutschen Profifußball, die auch berechtigterweise ein großes Medienecho hervorrief. Gleichzeitig ist aber festzustellen, dass ähnliche Geschehnisse im Amateurbereich häufiger auftreten und regelmäßig die zuständigen Sportgerichte beschäftigen: etwa in Form von Schiedsrichtern, die sich nach dem Abpfiff im Heizungskeller verstecken müssen, gebrochenen Nasen und Tumulten auf dem Fußballplatz. Auch wenn Täter und Opfer weniger prominent sind, lohnt sich eine wissenschaftliche Auseinandersetzung mit diesem Thema. Wer wird gegenüber wem warum auf dem Fußballplatz gewalttätig und lässt sich dies gegebenenfalls durch präventive Maßnahmen verhindern?

Diese forschungsleitende Frage steht im Mittelpunkt eines aktuellen Projekts am Institut für Kriminologie der Universität Tübingen in Zusammenarbeit mit dem Württembergischen Fußballverband e.V. (wfv) sowie mit Unterstützung des Deutschen Fußball-Bundes (DFB) und ist zugleich das Dissertationsthema der Verfasserin.

Im wfv sind rund 1800 Vereine mit über einer halben Million Mitglieder organisiert. Während der Spielzeit werden Wochenende für Wochenende etwa 5000 Spiele absolviert. Im Vergleich hierzu finden im reinen Profibereich der Männer (also der ersten, zweiten und dritten Liga) gerade einmal 28 Partien je Wochenende statt ${ }^{1}$. Alleine durch die Größe des Verbandsgebiets und die An- zahl der organisierten Vereine überrascht es also nicht, dass der wfv häufiger mit Gewaltvorkommnissen auf dem Fußballplatz konfrontiert wird als der DFB beziehungsweise die Deutsche Fußball Liga (DFL). Jedoch ist nicht nur die reine Anzahl der Spiele entscheidend. Grob lässt sich konstatieren, dass die Häufigkeit von Gewaltvorkommnissen abnimmt, je höher die Spielklasse ist. Mit steigender Professionalität der jeweiligen Liga steht für die Beteiligten im wahrsten Sinne des Wortes mehr auf dem Spiel, eine Sperrstrafe bedeutet dann nicht nur einen Einschnitt in die Freizeitgestaltung, sondern unter Umständen (teils massive) finanzielle Einbußen durch eine temporäre „Erwerbsunfähigkeit“. Jedoch sollte dieser Abschreckungseffekt nicht überschätzt werden. Für die geringere Anzahl von Gewaltvorkommnissen dürfte ein anderer Grund mitverantwortlich sein: mit zunehmendem Professionalisierungsgrad des Spielbetriebs verringern sich auch die Möglichkeiten zur Tatbegehung ${ }^{2}$.

Trotz der proportional höheren Belastung an Gewaltvorkommnissen der unteren Ligen haben Attacken auf den Schiedsrichter ${ }^{3}$ und vergleichbare Vorfälle gemessen an der Gesamtzahl aller Spiele eher Seltenheitscharakter. Erfreulicherweise läuft nach wie vor der größte Teil aller Spiele friedlich ab. Dennoch waren die Einzelvorfälle der letzten Jahre häufig von solcher Intensität, dass der Verband einen akuten Handlungsbedarf sah und in der Folge neue Präventionsmaßnahmen verabschiedete und sich um eine wissenschaftliche Aufarbeitung der Thematik bemühte.

\section{Was ist Gewalt, was nicht?}

Für die wissenschaftliche Beschäftigung mit dem Thema „Fußballgewalt“ war zunächst eine Definition erforderlich, welche gewalttätige Handlungen betrachtet werden sollen. Aktiv betriebener Sport nimmt hinsichtlich aggressiven Verhaltens immer eine Doppelrolle ein, „einerseits ist er Ventil für Kraftentfaltung und Erlebnisse des Erregungsauf- und -abbaus, andererseits
Aggressionsauslöser und -anreiz“ (Schwind et al. 1990: 96). Dies trifft für die Sportart Fußball in besonderem Maße zu, zumal eine große Wirkung der Zuschauer auf die Spieler - und umgekehrt - ihr Übriges tut und diesen Umstand häufig noch verstärkt. Wann ist aggressives Verhalten noch tolerierbares Wettkampfverhalten, wann ist die Grenze zur Gewalt überschritten?

Der Begriff der Gewalt lässt viel Interpretationsspielraum, was sich auch an den ausschweifenden Diskussionen unterschiedlichster Disziplinen zeigt. Obgleich insbesondere die ausgedehnte Auseinandersetzung mit dem Gewaltbegriff in der Soziologie durchaus seine Berechtigung hat, soll an dieser Stelle die Unbestimmtheit eingegrenzt werden, indem eine Konzentration auf kriminalisierte Gewalt erfolgt, zumal das Rechtssystem als soziales System „den Gewaltbegriff tagtäglich verbindlich auf konkrete soziale Sachverhalte anwendet" (Gemünden 2003: 334).

Ausgangspunkt der Untersuchung sind daher Sportgerichtsverfahren. Hierdurch sind sämtliche denkbaren Personenkonstellationen erfasst: Spieler, Schiedsrichter, Schiedsrichterassistenten, Trainer, sonstige Vereinsverantwortliche und weitere mittelbar an einem Fußballspiel Beteiligte (z.B. Ordner, Sanitäter, vereinseigene Linienrichter) können sowohl als Täter ebenso wie als Opfer auftreten. Auch Gewalt von $\mathrm{Zu}$ schauern (oder auch gegen Zuschauer) wird in Sportgerichtsverfahren verhandelt, sofern sie Einfluss auf das Spielgeschehen nimmt beziehungsweise Niederschlag im Spielbericht findet. Selbst in den Fällen, in denen die Zuschauer keine Vereinsmitglieder sind, sind die Sportgerichte zuständig und die Verbandsautonomie wirkt durch die Vereinshaftung. Schlagen sich jedoch beispielsweise ausschließlich „Fans“ vor oder nach einem Spiel ohne Auswirkung auf die Partie, werden die Sportgerichte mangels Zuständigkeit zumeist nicht tätig. Die typische „Hooliganproblematik“ ist somit nicht Gegenstand dieser wissenschaftlichen Aufarbeitung (s. dazu Kett-Straub in diesem 
Heft); sie spielt ohnehin im (württembergischen) Amateurbereich kaum eine Rolle.

Trotz des Ausklammerns dieser Personengruppe bleiben genügend Divergenzen hinsichtlich der Definition von gewalttätigem Verhalten. Unter Spielern sind während des Spiels körperliche Auseinandersetzungen bzw. Aggressionen in einem gewissen Ausmaß spielimmanent und eher tolerierbar, gegenüber dem Schiedsrichter und anderen Dritten sind diese den Spielordnungen nach gänzlich verboten. Daher sind Gewalt und gewalttätiges Verhalten gegenüber diesen Personen leichter zu umreißen. „Klassische“ gewalttätige Verhaltensweisen werden in den Rechtsordnungen der Fußballverbände in der Hauptsache unter dem Rechtsbegriff der Tätlichkeit (intentionales, körperlich-gewaltsames Vorgehen gegen andere Spielbeteiligte; vgl. Hilpert 2009: 124) aufgeführt. Wenngleich vom engen Gewaltbegriff nicht umfasst (Gewalt als körperlich wirkender Zwang), erscheint darüber hinaus die Einbeziehung von Beleidigungen und Bedrohungen in die Untersuchung als sinnvoll. Zudem sind bestimmte Formen unsportlichen Verhaltens (etwa körperliche Übergriffe außerhalb des eigentlichen Spielgeschehens, aber unterhalb der Schwelle zur Tätlichkeit oder Gewalt gegen Sachen im Zusammenhang mit gewalttätigem Verhalten gegen Schiedsrichter) zu berücksichtigen.

Zugleich ist aber auch - abgesehen von den Tätlichkeiten - eine gewisse Intensität beziehungsweise Schwere der Vergehen erforderlich; eine einfache Beleidigung wie „Schiri, Du bist blöd!“ ist zwar streng genommen auch verbale Gewalt, gehört aber nicht zur Kernproblematik. So sieht der wfv insbesondere Tätlichkeiten, Beleidigungen, Bedrohungen und unsportliches Verhalten $a b$ einer individuellen Spielsperre von drei Monaten als schwere Vergehen an. Dieses Konstrukt dient als Ausgangspunkt für die hier untersuchten Gewaltvorkommnisse. Juristisch betrachtet stehen damit solche Verhaltensweisen im Zentrum, die in ihrer Mehrzahl nicht nur vereinsdisziplinarrechtliche Konsequenzen zeitigen können, sondern zugleich allgemein strafrechtlich relevant sind; aus strafrechtlicher Warte stehen Beleidigungs-, Nötigungs-, Bedrohungsdelikte $\left(\mathbb{1} 185 \mathrm{StGB} ; \mathbb{\int} \int 240 \mathrm{f}\right.$. StGB) sowie vorsätzliche Körperverletzungsdelikte ( $\mathbb{S} 223 \mathrm{ff}$. StGB) im Fokus.

Bei der Auseinandersetzung mit Gewalt im Fußball sind neben diesen Tatbeständen zwingend auch Spielabbrüche zu berück- sichtigen ${ }^{4}$. Der Spielabbruch stellt den worst case im Fußball dar. Der Schiedsrichter ist nach Anweisungen des DFB gehalten, alle zumutbaren Mittel auszuschöpfen, um ein Spiel regulär zu beenden. Dieser Anweisung kam auch der Schiedsrichter Wolfgang Stark im eingangs erwähnten Skandalspiel nach und wartete lange 21 Minuten ab, um dann das Spiel nochmals für 93 Sekunden anzupfeifen.

\section{Ausgangssituation}

Die Abteilung Recht des wfv zählte in der Saison 2009/2010 388 schwere Gewaltvergehen, in der folgenden Saison 2010/2011 waren es 347. Darunter waren 2009/2010 61 Spielabbrüche, 2010/2011 reduzierte sich die Zahl auf 43 nicht regulär beendete Partien. Auch in den vorangegangenen Spielzeiten gab es immer wieder bestürzende Vorfälle, die für viel Aufsehen sorgten.

Bereits in der Vergangenheit hat sich der wfv in Kooperation mit dem Landeskriminalamt Baden-Württemberg bemüht, Präventionsmaßnahmen voranzutreiben, um die damals feststellbare Zunahme von Gewaltvorkommnisse einzudämmen. Durch unterschiedliche Module der Aktion „wfv für Toleranz und Fairness - gegen Gewalt“ wurde versucht, die positiven Elemente des Fußballs zu fördern und gleichzeitig negative Einflüsse zu verringern (vgl. Ehmig/ Röchling 2006). Trotz allen Engagements reichten die Bemühungen jedoch nicht aus, um eine grundlegende Trendwende zu erreichen.

Daher wurden zu Beginn der Spielzeit 2010/2011 mehrere Änderungen präventiven Charakters in der Spielordnung und den Durchführungsbestimmungen verankert. Jenseits der bloßen Reaktion auf Gewalthandlungen im Fußball durch die Sportgerichte war der Verband bestrebt, neben den bestehenden Konzepten und Strategien neue Präventionsmaßnahmen zur Eindämmung von Gewaltvorkommnissen zu installieren. Zum einen galt es (potentielle) Opfer zu schützen, zum anderen sollte der Fußball wieder verstärkt als persönlichkeitsbildendes Spiel in den Mittelpunkt rücken. Als Teil eines „Maßnahmenpakets zur Gewaltprävention und Deeskalation" beschloss der Vorstand des wfv am 04.06.2010 wegen Dringlichkeit vorläufige Ordnungsänderungen, mit deren Hilfe sich Gewalt vermeiden oder ablaufende Eskalationsprozesse zumindest unterbrechen lassen sollen. Durch die Veränderung von Rand- bedingungen soll der Ablauf eines Fußballspiels risikoärmer werden. Von besonderer Bedeutung sind hierbei das Einrichten einer Technischen Zone und die Bereitstellung gekennzeichneter Platzordner durch die Heimvereine. Diese Ordnungsänderungen traten am 01.07.2010 zu Beginn des neuen Spieljahres in Kraft; ebenso wie eine Änderung der Durchführungsbestimmungen, die zur Einführung des Handschlags vor dem Spiel führte . $^{5}$.

Diese neuen Maßnahmen wurden - neben anderen Komponenten - im Besonderen bei der durchgeführten Studie, die im Folgenden vorgestellt wird, berücksichtigt und beleuchtet.

\section{Konzeption der Untersuchung}

In einer Vollerhebung werden alle Sportgerichtsurteile einschließlich eventueller Berufungsverfahren (sämtlicher Spiel- und Altersklassen) im Zuständigkeitsgebiet des wfv erhoben, die schwere Vergehen ab einer Sperrzeit von drei Monaten sowie unabhängig vom Strafmaß Spielabbrüche zum Gegenstand haben. Dabei werden zwei Spielzeiten berücksichtigt: zum einen die Saison 2009/2010 vor den Ordnungsänderungen, zum anderen die Saison 2010/2011 danach.

Für die Aktenauswertung der Sportgerichtsurteile wurde ein umfangreiches, zwanzigseitiges Analyseraster entwickelt, in dem mehr als 130 Variablen rund um die einzelnen Gewaltvorkommnisse abgefragt werden. Hiermit können die typischen Erscheinungsformen gewalttätiger Verhaltensweisen auf dem Fußballplatz (äußeres Erscheinungsbild, Schwere der Tat sowie ihre Konsequenzen, z.B. Beeinträchtigung des Opfers) erfasst werden, darüber hinaus werden Ursachen, Auslöser und individuelle Motivlagen von Gewalt berücksichtigt, sofern sich diese rekonstruieren lassen. Ebenso werden weitere wichtige Faktoren bezüglich Tätern und Opfern einbezogen wie etwa Alter, Geschlecht, ethnische und regionale Herkunft, „Professionalisierungsgrad“ der Schiedsrichter und Spieler (Erfahrung, Spielklasse etc.) sowie alle Informationen rund um das Spiel (Ergebnisse, allgemeiner Spielaufbau, Tabellenkonstellation der beiden Mannschaften etc. $)^{6}$. So werden relevante Informationen zu den strukturellen sowie individuellen Bedingungen, die im Zusammenhang mit dem jeweiligen Gewaltvorkommnis stehen, herausgearbeitet, um identifizieren zu können, in welchen Situationen beziehungsweise Konstellationen es 
besonders häufig zu Gewalt auf dem Fußballplatz kommt.

Zwischen den beiden Spielzeiten, die untersucht werden, kam es zu einem unübersehbaren Rückgang der Vorfälle (-12\%). Da die Aktenanalyse noch nicht abgeschlossen ist, lassen sich jedoch noch keine Aussagen darüber treffen, ob dies einer zufälligen Schwankung geschuldet ist, oder ob die Maßnahmen - wie erhofft - bereits gegriffen haben.

Ein weiterer Projektteil ist hingegen schon abgeschlossen: im Spätherbst 2011 wurde eine umfassende Umfrage unter allen württembergischen Schiedsrichtern durchgeführt, um zusätzliche Aspekte zu beleuchten, die durch die Sportgerichtsaktenanalyse nicht gewonnen werden können. Es liegt gerade auch im Interesse des Verbands zu erfahren, was die Schiedsrichter auf württembergischen Fußballplätzen tatsächlich erleben, und zwar unabhängig davon, ob diese Vorfälle von der Sportgerichtsbarkeit zur Kenntnis genommen wurden oder nicht. Durch den Einsatz des Fragebogens ist es möglich, dies aus unmittelbarer Perspektive in Erfahrung zu bringen. Dies ist umso interessanter, da sich Untersuchungen zu Gewaltvorkommnissen im Fußball bislang zumeist auf Analysen von Sportgerichtsurteilen und damit nur auf „Hellfeld“-Daten stützen (z.B. Scherer/Winands 2010, Stahl 2010, Pulter et al. 2006). In allen 41 württembergischen Schiedsrichtergruppen wurde die Umfrage in den normalen Schiedsrichter-Schulungsbetrieb integriert. Insgesamt konnten so Informationen von 2602 Schiedsrichtern gewonnen werden.

Die Unparteiischen wurden gebeten, einen vierseitigen Fragebogen auszufüllen. Zum einen wurden sie gefragt, wie sie selbst die Konzeption und die Wirkung der neuen Regelungen bewerten, zum anderen sollten sie ihr persönliches Sicherheitsgefühl einschätzen. Abgerundet wurde die Umfrage mit einigen Fragen zum „Dunkelfeld“ beziehungsweise ihrem eigenen Meldeverhalten bezüglich Beleidigungen, Bedrohungen und tätlicher Angriffe, daneben wurden einige persönliche Angaben (Staatsangehörigkeit, Geburtsjahr, höchster allgemeiner Bildungsabschluss) und Angaben zur Schiedsrichtertätigkeit (Schiedsrichtererfahrung, Qualifikation/Spielklasse, Anzahl der übernommenen Spielleitungen in der letzten Saison) erfasst.
Ein Teil der Ergebnisse, die die neuen Präventionsmaßnahmen zum Inhalt haben, wird im Folgenden vorgestellt.

\section{Einrichten der Technischen Zone}

Der $\mathbb{4} 44$ Abs. 2 der wfv-Spielordnung wurde dahingehend ergänzt, dass eine Technische Zone einzurichten ist ${ }^{7}$. Vor der Spielzeit 2010/2011 war eine Coaching-Zone dieser Art nur von der Bundes- bis zur Oberliga üblich, nicht aber im unteren Amateurbereich. Die Einführung dieser neuen Regel stieß nicht überall auf Verständnis, nicht wenige Vereinsvertreter hielten einen markierten Bereich am Spielfeldrand für überflüssig ${ }^{8}$; die Technische Zone schien für diese Personengruppe eher ein lästiges Übel zu sein. Um zu erfahren, ob die Schiedsrichter dies genauso beurteilen, wurden sie gebeten, ihre Meinung zu äußern, ob sie die Einführung dieser Regelung für sinnvoll erachten oder nicht.

\section{Abbildung 1: „Das Einrichten einer} Technischen Zone halte ich für eine sinnvolle Idee."

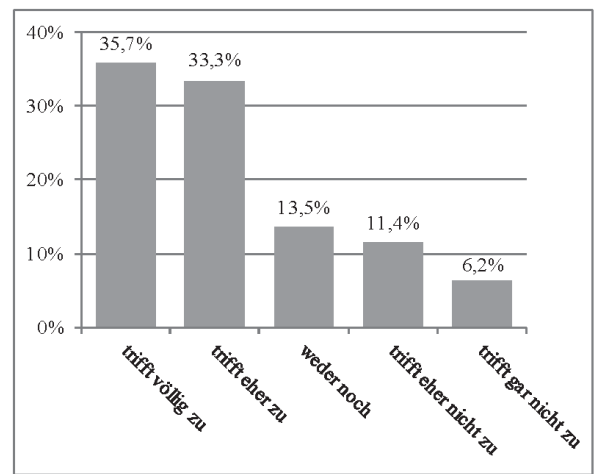

Wie sich Abbildung 1 entnehmen lässt, ist die Zustimmung unter den Schiedsrichtern recht groß; 69,0 Prozent halten die Technische Zone für eine positive Neuerung. Somit halten weit mehr als zwei Drittel derjenigen Personen, deren Schutz die Technische Zone - neben der Erleichterung für die allgemeine Spielkontrolle - hauptsächlich dienen soll, diese für ein taugliches Mittel. Dies dürfte nicht nur für den Verband ein sehr zufriedenstellendes Ergebnis darstellen.

\section{Handschlag vor dem Spiel}

Des Weiteren wurde zur Förderung des Fair-Play-Gedankens unter dem Punkt 7a der Durchführungsbestimmungen für die Verbandsspiele im Spieljahr 2010/2011 der Handschlag vor dem Spiel eingeführt. Der Handschlag hat zwischen den Spielern beider Mannschaften sowie dem Schiedsrichter zu erfolgen ${ }^{9}$. Die Schiedsrichter wurden gefragt, wie sie diese Regelung beurteilen.

Abbildung 2: „Den Handschlag vor dem Spiel halte ich für eine sinnvolle Idee. "

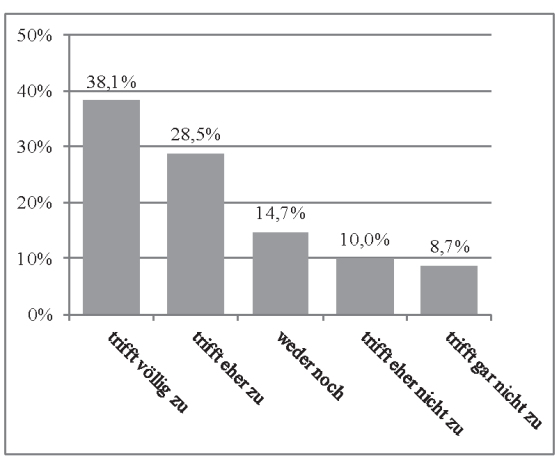

Auch hier zeigt sich eine breite Zustimmung zur neuen Regelung, 66,6 Prozent der Schiedsrichter stehen ihr positiv gegenüber, wobei 14,7 Prozent keine Wertung dazu abgeben. Dennoch hält knapp jeder fünfte Schiedsrichter (18,7\%) den Handschlag vor dem Spiel für eher weniger oder überhaupt nicht sinnvoll. Weitgehend ungeklärt muss dabei bleiben, ob diese Ablehnung genereller Natur ist oder eventuell daher rührt, dass die Schiedsrichter sich eine andere Ausgestaltung der Regelung gewünscht hätten.

Dies ist vor allem deshalb denkbar, da nicht nur die Verbandsverantwortlichen vor der Einführung des Handschlags darüber diskutierten, ob der Handschlag ausschließlich vor oder zusätzlich auch nach dem Spiel zur Pflicht werden sollte. Letztlich wurde auf den Handschlag nach dem Spiel verzichtet, da man befürchtete, dass eventuell erst durch die Neuregelung Aggressionen provoziert werden könnten, also wenn Spieler etwa nach einer bitteren Niederlage dem Gegner „auch noch“ die Hand geben müssen.

Diese Gedankenspiele griffen manche Schiedsrichter auf, die handschriftlich auf dem Fragebogen vermerkten, dass sie es sehr begrüßt hätten, wenn auch der Handschlag nach dem Spiel verpflichtend gewesen wäre. Anders als die obige Argumentation sind sie der Meinung, dass gerade aus diesem Grund der Handschlag nach dem Spiel - also quasi die Pflicht zur Gratulation - geeignet wäre, um die Spieler noch mehr auf den Fair-PlayGedanken zu „trimmen“.

\section{Kennzeichnungspflicht der Ordner}

Die wohl umfassendste und strittigste Ordnungsänderung stellt die Kennzeichnungspflicht der Ordner dar. Dabei ist die Pflicht zur Ordnerstellung keineswegs neu, 
nur wurde diese durch die Benennung und Kennzeichnung augenscheinlicher und kontrollierbarer beziehungsweise wurde ihr mehr Nachdruck verliehen. Durch die Mindestanzahl von zwei Ordnern ist nun die Diskussion obsolet, ob ein Bedarf an Ordnungskräften besteht oder nicht, wie sich der Neufassung des $\$ 36$ a der wfv-Spielordnung entnehmen lässt:

Platzordner müssen bei Bedarf in genügender Anzahl aufgeboten werden und sind mit Signalwesten kenntlich zu machen. Bei Verbands- und Verbandspokalspielen der Herren sind vom Platzverein mindestens zwei durch Signalwesten gekennzeichnete und vor dem Spiel auf dem Spielberichtsbogen namentlich zu benennende Ordner zu stellen. Dies gilt auch für Verbands- und Verbandspokalspiele der Reserve, der A- und B-Junioren sowie der Frauen, es sei denn, eine Gefährdung der Ordnung und Sicherheit ist offenkundig nicht gegeben und der Schiedsrichter besteht nicht ausdrücklich auf einer Gestellung. Den Gastvereinen wird empfohlen, bei Verbands- und Verbandspokalspielen einen Ansprechpartner für Ordnung und Sicherheit zu stellen, der durch eine Armbinde mit der Aufschrift „Gast“ gekennzeichnet ist.

Diese Regelung, die auch für die Ausrichter von Hallen- und Turnierspielen gilt, wurde ebenso wie das Einrichten der Technischen Zone eher mit negativen Emotionen seitens der Vereine bedacht. Dabei war häufig von „Kanonen auf Spatzen“ die Rede, insbesondere wurde der organisatorische Aufwand für zu groß erachtet ${ }^{10}$. Auch hier wurden wiederum die Unparteiischen gefragt, ob sie die neue Maßnahme für sinnvoll erachten oder nicht.

Abbildung 3: „Die Kennzeichnung der Ordner (durch Tragen der Weste) halte ich für eine sinnvolle Idee. "

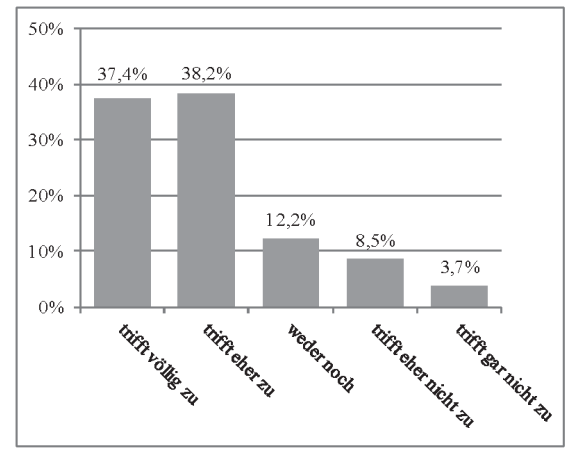

Es zeigt sich eine außerordentlich hohe $\mathrm{Zu}$ stimmung: mehr als drei Viertel der Schiedsrichter $(75,6 \%)$ beurteilen die Kennzeich- nungspflicht der Ordner wohlwollend. Damit liegen die zustimmenden Werte auch noch deutlich über denen der beiden anderen Maßnahmen, obwohl ja auch diese bereits von einer breiten Mehrheit der Schiedsrichter positiv beschieden wurden.

Die Diskussion rund um das Thema Ordnungskräfte ist damit jedoch noch längst nicht erschöpft. Zusätzlich wurden die Unparteiischen gefragt, welche Erfahrungen sie bislang mit den Ordnungskräften machten. Auf die Frage „Haben Sie schon einmal einen Ordner-Einsatz in Anspruch genommen, um für Ruhe oder Sicherheit auf oder neben dem Platz zu sorgen?" antworteten 25,9 Prozent der Schiedsrichter $(\mathrm{n}=548)$ mit „ja“. Folglich ist der Bedarf an Ordnungskräften durchaus gegeben; ganz abgesehen davon, wie wichtig es für das Sicherheitsgefühl eines Schiedsrichters sein kann, zu wissen, dass im Ernstfall mit Hilfe vom Spielfeldrand zu rechnen ist.

$\mathrm{Ob}$ es in solch einem Ernstfall ausreichend ist, dass überhaupt jemand zur Verfügung steht, oder ob es auch eine Rolle spielt, wer diese Aufgabe übernimmt, steht auf einem anderen Blatt. Daher wurde den Schiedsrichtern ferner folgende Frage gestellt: „Sind Sie grundsätzlich mit den Personen, die die Vereine als Ordner stellen, zufrieden?". Dies bejahten 82,1 Prozent der Befragten. Im Großen und Ganzen sind die meisten Unparteiischen demnach zufrieden mit den Personen, die von den Vereinen als Ordner gestellt werden. Die 17,9 Prozent, die angaben, dass sie grundsätzlich unzufrieden mit der Personenauswahl sind, wurden zusätzlich in einer offenen Variablen nach den Gründen für ihr Missfallen gefragt. Auf diese soll im Folgenden genauer eingegangen werden. Nach einer Kategorisierung ergibt sich folgendes Bild:

Tabelle 1: Gründe für die generelle Unzufriedenheit bei der Ordnerauswahl

\begin{tabular}{|l|r|}
\hline Parteilichkeit bzw. „Vereinsbrille“: & $16,5 \%$ \\
\hline $\begin{array}{l}\text { eigener Alkoholkonsum der } \\
\text { Ordner: }\end{array}$ & $5,5 \%$ \\
\hline mangelnde Ernsthaftigkeit: & $23,2 \%$ \\
\hline unzureichende Schulung: & $17,4 \%$ \\
\hline $\begin{array}{l}\text { Ungeeignetheit (ohne Konkreti- } \\
\text { sierung): }\end{array}$ & $10,4 \%$ \\
\hline $\begin{array}{l}\text { Abwesenheit der Ordner/ } \\
\text { unzureichende Kennzeichnung: }\end{array}$ & $14,3 \%$ \\
\hline Alter der Ordnungskräfte: & $5,2 \%$ \\
\hline aggressives Verhalten: & $6,4 \%$ \\
\hline Sonstiger Grund: & $1,2 \%$ \\
\hline
\end{tabular}

Im Großen und Ganzen dominiert bei der ablehnenden Haltung gegenüber den ausgewählten Personen der Umstand, dass die Schiedsrichter den Eindruck haben, der Ordnerdienst werde von diesen nicht ernst genommen (,irgendwelche Abnungslosen, wo keine Lust haben, das Amt auszuüben", „viele tragen nur ihre Weste und sehen das als getane Arbeit“, „oft kommen Notlösungen zum Einsatz“, „zumeist reine Alibifunktion“, „gezwungene Ordner mit Desinteresse", „sie tragen die Weste nur, weil es Vorschrift ist", „halten das Ganze für ein Kaschperletheater!"); immerhin fast jeder vierte unzufriedene Unparteiische $(23,2 \%)$ nannte dies als ausschlaggebenden Grund.

Am zweithäufigsten wurde angemahnt, dass die Personen, die den Ordnerdienst übernehmen, nur unzureichend geschult seien (17,4 \%). Die ausgewählten Personen wüssten nicht Bescheid, was zu ihren Aufgaben zählt und wie sie sich zu verhalten haben („,die Ordner sind nicht ausgebildet“, „zum Großteil kommen inkompetente und desinteressierte Personen zum Einsatz“, „die nötige Ausbildung fehlt“, „zu wenig geschult, unprofihaft, meistens Witzfiguren“,,es fehlt oft an der Eignung zur Deeskalation beizutragen“, „willkürlich ausgewählte Personen obne Kenntnisse“, „weil sie meistens nicht auf Konflikte geschult sind").

An dritter Stelle mit 16,5 Prozent wurde die Ungeeignetheit aufgrund von Parteilichkeit der Personen genannt (etwa „die Ordner sind immer für den eigenen Verein“, „diese verfolgen den Spielverlauf zu sehr durch die Vereinsbrille“, „es sind meistens eingefleischte Fans", „die Ordner sind oft zu befangen“, „meist emotional sehr stark mit dem Verein verbundene Personen" oder auch „diese wahren die gewünschte Neutralität nicht").

Ein weiterer Teil der Schiedsrichter (14,3\%) kritisiert, dass die Ordner schlichtweg nicht vorhanden oder nicht zu erkennen sind. Dabei kam sowohl die dauerhafte Abwesenheit (, ,habe noch nie eine Person mit Ordnerweste gesehen bzw. war noch nie vorhanden", „meistens ist niemand da bzw. steht kein Ordner zur Verfügung“, „Ordner werden selten eingetragen, der SR muss oft nachfragen!") als auch die temporäre Abwesenheit vom Spielfeldrand (,bevorzugter Standort der Ordner: Würstchenstand und/oder Getränkebude“, „sie sind für den SR nicht greifbar, wenn es zu Auseinandersetzungen kommen würde!") zur Sprache. Selbst wenn die Ordner (irgendwo) vor Ort sind, kann der Schiedsrichter nicht immer auf die Ord- 
ner zurückgreifen, wenn der Kennzeichnungspflicht nicht ordnungsgemäß nachgekommen wird (, man sieht sie meistens gar nicht, erst wenn es drauf ankommt, ist es manchmal schon zu spät", „sind selten vorstellig oder gekennzeichnet").

Jeder zehnte unzufriedene Schiedsrichter $(10,4 \%)$ bemängelt die Ordnerauswahl, jedoch ohne genauer zu konkretisieren, warum die Personen ungeeignet für den Ordnerdienst seien („es werden ungeeignete Personen ausgewählt", ,teilweise unseriöses Auftreten“, „sehr oft werden einfach total unfähige Personen zur Verfügung gestellt! ", „lustlos, nicht geeignet“, „die Auswabl der Ordner ist eher fragwürdig").

Unerfreulicherweise berichten Schiedsrichter $(6,4 \%)$ auch von einer zugespitzten Form von Parteilichkeit der Ordner, und zwar dergestalt, dass diese selbst zu aggressivem Verhalten neigen (,einige der Ordner meckern und schreien zusammen mit den Zuschauern", „meistens die größten Unruhestifter", "diese beschimpfen den Schiri auch", "teilweise sind die Ordner an Eskalationen mit- oder hauptverantwortlich ", „diese reklamieren meist selbst!“, „viele Ordner schreien am lautesten!").

Einen weiteren Kritikpunkt stellt der Alkoholkonsum der Ordner dar, der von 5,5 Prozent der unzufriedenen Schiedsrichter vorgebracht wurde (,sie missbilligen die Tätigkeit z.B. durch Genuss von Alkohol", „die Ordner saufen (fast alle) “, „auch diese konsumieren oft während dem Spiel Alkohol", „die rauchen und saufen und sind weit von dem Geschehen weg “, „weil sie alkoholische Getränke zu sich nehmen und selber lautstark gegen den SR sind“, „fübren oft Ordnertätigkeit unter Alkohol durch").

Auch wurde das Alter der Ordnungskräfte von einigen Befragten (5,2\%) beanstandet. Dabei werden die Ordner teils als zu alt, teils aber auch als zu jung angesehen („,es sind oft Rentner oder Leute, die sich nicht durchsetzen können“, „20jährige oder Frauen“, „es sind oft auch alte oder sehr junge Ordner", „es sind überwiegend ältere Ordner, die keine Sicherheit geben, da sie nicht in der Lage sind bei jungen Zuschauern für Rube zu sorgen“, „oft zu jung und dementsprechend unerfahren im Umgang mit Konflikten"). Verschwindend gering war die Nennung sonstiger Gründe mit 1,2 Prozent (beispielsweise „diese Personen sollten stärker gebaut sein").

So bleibt festzuhalten, dass die Schiedsrichter teils mit der Umsetzung bzw. Durch- führung der Neuregelung unzufrieden sind (also etwa der Kennzeichnung der Ordner) und teils die hierfür ausgewählten Personen - aus den unterschiedlichsten Gründen - für ungeeignet halten. Auch wenn die Begründungen streckenweise kein gutes Licht auf die Vereine werfen, ist zu bedenken, dass diese Kritik nur von einem kleineren Teil der Schiedsrichter geäußert wurde. Die Mehrzahl der Unparteiischen ist demnach zufrieden mit den zur Verfügung gestellten Ordnern, so dass festzuhalten ist, dass sich die meisten Vereine durchaus bemühen, die neuen Bestimmungen sachgerecht zu erfüllen.

Trotzdem scheint - zumindest stellenweise - eine Diskrepanz zwischen Theorie und Praxis zu herrschen. So befindet eine überwältigende Mehrheit der befragten Schiedsrichter die neuen Regelungen, insbesondere die Kennzeichnungspflicht der Ordner, für sinnvoll. Gleichzeitig deutet die Frage nach der Zufriedenheit mit den Ordern darauf hin, dass die Umsetzung der Regelung noch verbesserungswürdig ist. Daher wurden die Schiedsrichter zusätzlich gebeten, einzuschätzen, inwieweit die drei Neuregelungen tatsächlich angewandt werden, ob diese doch eher unnötig sind und ob sich die Neuerungen derart in der Praxis bewährt haben, dass man davon sprechen kann, dass sie die Sicherheit auf den württembergischen Fußballplätzen merklich verbessert haben.

In Anlehnung an die Kritik der Vereine - sowie als zusätzliche Kontrollmöglichkeit für die Fragen nach der Sinnigkeit der einzelnen Maßnahmen - sollten die Unparteiischen zunächst beurteilen, ob die Ordnungsänderungen überhaupt notwendig waren.

Abbildung 4: „Die neuen Regelungen sind überflüssig."

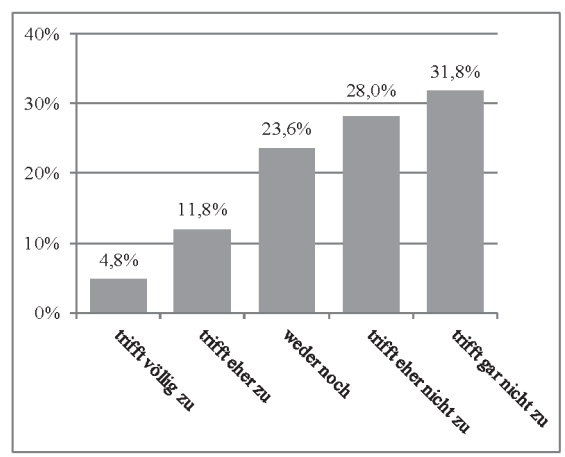

16,6 Prozent der Schiedsrichter vertreten demnach die Meinung, dass es der neuen Regelungen nicht bedarf, knapp ein Viertel $(23,6 \%)$ ist diesbezüglich unentschlossen. Mit 59,8 Prozent sieht jedoch der Großteil der Befragten durchaus eine Daseinsberech- tigung für die neuen Regelungen im Gesamten. Eng verknüpft mit der Frage nach der grundsätzlichen Überflüssigkeit ist auch die Überlegung, inwieweit die Regelungen in der Praxis überhaupt angekommen sind und Wirkung zeigen.

Abbildung 5: „Die neuen Regelungen des wfv zur Saison 2010/2011 haben die Sicherheit auf den Plätzen spürbar verbessert."

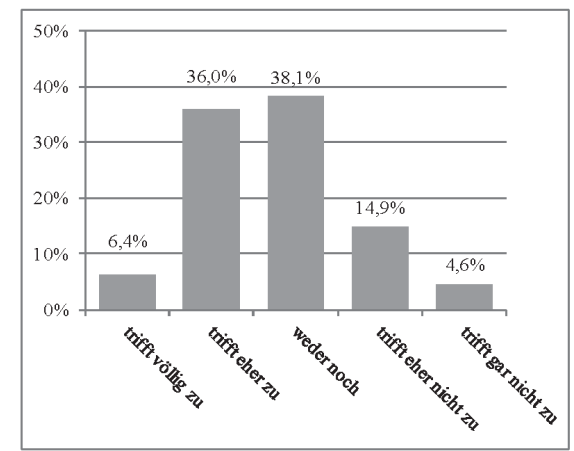

Immerhin 42,4 Prozent nehmen eine spürbare Verbesserung wahr. Dies ist einerseits positiv zu werten, da die neuen Präventionsmaßnahmen durchaus Erfolge zu erzielen scheinen. Gleichzeitig ist jedoch die Mehrzahl der Schiedsrichter der Meinung, dass keine nennenswerten Veränderungen eingetreten sind. Dieser Befund könnte sich auf mehrere Ursachen zurückführen lassen. So ist etwa denkbar, dass die Maßnahmen noch nicht wirken, da sie noch nicht richtig implementiert wurden. So gab es vor allem in der Anfangszeit teilweise Probleme mit den Ordnerwesten, da nicht allen Vereinen rechtzeitig zu Beginn der Saison wie angekündigt zwei Signalwesten zur Verfügung gestellt werden konnten. In der Folge gab es auch eine Übergangszeit, in der die Nichtbefolgung der neuen Regelungen keine Bestrafung nach sich zog. Zum anderen ist auch vorstellbar, dass ein Teil der Schiedsrichter eine Verbesserung der Sicherheitslage gar nicht für notwendig oder möglich hält, da ihrer Meinung nach kein Gefahrenpotential vorliegt, etwa in nur wenig mit Gewaltvorkommnissen belasteten Bezirken. Dies gilt es zu klären, wenn sämtliche Daten vorliegen und damit auch Abgleiche zwischen dem Aktenmaterial und den Umfrageergebnissen möglich sind.

\section{Vorläufiges Fazit}

Der Württembergische Fußballverband installierte im Sommer 2010 neue Präventionsmaßnahmen, um Gewaltvorkommnisse im Verbandsgebiet zu reduzieren. In den Jahren zuvor setzte der Verband eher auf 
Kampagnen allgemeiner Natur, die sich pauschal an alle richten und der positiven Generalprävention zuzurechnen sind, wie beispielsweise die Aktion „Fair ist mehr!“. Die neuen Maßnahmen hingegen richten sich mitunter an potentielle Täter und sind daher der selektiven beziehungsweise sekundären Prävention zuzuschreiben (vgl. dazu Schwind 2011: 20). Dabei wurde ein konkretes Gefährdungspotential bereits identifiziert $^{12}$, eine mögliche Wirksamkeit lässt sich dadurch einfacher evaluieren.

Die Aktenanalyse wird wichtige Hinweise liefern, ob sich der Rückgang der Gewaltvorkommnisse zwischen 2009/2010 und 2010/2011 auf das Maßnahmenpaket, das bei den Mitgliedsvereinen höchst umstritten war, zurückführen lässt. Unabhängig von diesen Ergebnissen lässt sich aber bereits jetzt festhalten, dass die Einführung dieser Maßnahmen als Erfolg gewertet werden kann: die Schiedsrichter, zu deren Schutz die neuen Regelungen unter anderem gedacht sind, erachten sie für sinnvoll und haben bei einem Teil der Befragten dazu beigetragen, dass sich das (subjektive) Sicherheitsempfinden verbesserte. Gerade bei den Schiedsrichtern in den unteren Ligen, in denen das Ehrenamt nur geringfügig beziehungsweise symbolisch entschädigt wird ${ }^{13}$, ist es von großer Wichtigkeit, dass sich die Schiedsrichter sicher fühlen, um weiterhin gerne in ihrer Freizeit das Amt des Spielleiters auszuüben.

\section{Kontakt}

Thaya Vester, M.A.

Institut für Kriminologie der Universität Tübingen

Sand 7

72076 Tübingen

E-Mail: thaya.vester@uni-tuebingen.de

Ansprechpartner des Württembergischen Fußballverbands

David Biedemann

Württembergischer Fußballverband

Goethestraße 9

70174 Stuttgart

E-Mail: d.biedemann@wuerttfv.de

\section{Literatur}

Ehmig, Reinhold \& Röchling, Alexander (2006): wfv - für Toleranz und Fairness - gegen Gewalt. Aktionen des Württembergischen Fußballverbandes gegen Gewalt im Fußball in Kooperation mit dem Landeskriminalamt Baden-Württemberg. In: Forum Kriminalprävention, Heft 3, Seite 20-27

Gemünden, Jürgen (2003): Gewalt in Partnerschaften im Hell- und Dunkelfeld. Zur empirischen Relevanz der Gewalt gegen Männer. In: Lamnek, S./Boatcă, M. (Hrsg.):
Geschlecht - Gewalt - Gesellschaft. Opladen, Seite 333-353

Hilpert, Horst (2009): Das Fußballstrafrecht des Deutschen Fußball-Bundes (DFB): Kommentar zur Rechts- und Verfahrensordnung des Deutschen Fußball-Bundes (RuVO) nebst Erläuterungen von weiteren Rechtsbereichen des DFB, der FIFA, der UEFA, der Landesverbände. Berlin

Pulter, Astrid \& Pulter, Klaus \& Ribler, Angelika (2006): Ergebnisse der Evaluation von Rechtsurteilen des HFV. In: Ribler, Angelika \& Pulter, Astrid (Hrsg.): Konfliktmanagement im Fußball. Handbuch zum Projekt „Interkulturelle Konfliktvermittlung - Mediation im Fußball“ 1998-2005. Frankfurt, Seite 61-66

Scherer, Judith \& Winands, Martin (2010): Konfliktbelastungen im Amateurfußball. In: Ribler, Angelika \& Pulter, Astrid (Hrsg.): Konfliktmanagement im Fußball. Frankfurt, Seite 47-54

Schwind, Hans-Dieter \& Baumann, Jürgen \& Schneider, Ursula \& Winter, Manfred (1990): Gewalt in der Bundesrepublik Deutschland. Endgutachten der Unabhängigen Regierungskommission zur Verhinderung und Bekämpfung von Gewalt (Gewaltkommission). In: Schwind et al. (Hrsg.): Ursachen, Prävention und Kontrolle von Gewalt. Analysen und Vorschläge der Unabhängigen Regierungskommission zur Verhinderung und Bekämpfung von Gewalt (Gewaltkommission). Band I. Endgutachten und Zwischengutachten der Arbeitsgruppen. Berlin

Schwind, Hans-Dieter (2011): Kriminologie. Eine praxisorientierte Einführung mit Beispielen, 21., neubearbeitete u. erweiterte Auflage. Heidelberg

Stahl, Silvester (2010): Sportgerichtsurteile im Berliner Fußball-Verband 1999-2009. Potsdam

\section{Fußnoten:}

1 In der ersten und zweiten Bundesliga finden wöchentlich für gewöhnlich jeweils neun Partien, in der dritten Fußball-Liga zehn Partien statt.

2 In den unteren Ligen ist kein unabhängiges Schiedsrichtergespann tätig, der Schiedsrichter wird von „Vereinslinienrichtern“ unterstützt. Hat ein Schiedsrichter „eigene“ Assistenten zur Verfügung, dürfte er nicht zuletzt auch deshalb souveräner auftreten, da er weiß, dass er Unterstützung hat, auf die er sich verlassen kann. Zusätzlich gibt es, je höher die Spielklasse ist, weitere Anforderungen an die Sicherheit, also beispielsweise bestimmte Ordnungskräfte und bauliche Maßnahmen etc., die dazu beitragen, Gewaltvorkommnisse zu reduzieren.

3 Da der größte Teil der Schiedsrichter männlichen Geschlechts ist, wird zur Vermeidung der doppelten Nennung männlicher und weiblicher Formen die männliche Sprachform gewählt; dies dient ausschließlich der besseren Lesbarkeit.

4 Abgebrochen werden müssen Spiele vereinzelt auch aufgrund schlechter Witterung oder auch auf Wunsch der Mannschaftsführer, wenn die eigene Mannschaft durch Verletzungen oder rote Karten weniger als sieben Spieler auf dem Feld hat (vgl. $\mathbb{1} 1$ IV 2 der wfv-Spielordnung). Diese Spielabbrüche ohne Gewaltvorkommnisse wurden in der Analyse nicht berücksichtigt.

5 Im Spätsommer 2010 führte der Verband insgesamt 39 Ordnerschulungen durch, um die Vereine mit den neuen Regelungen vertraut zu machen.

6 Zusätzlich werden sämtliche Angaben rund um das Sportgerichtsverfahren erfasst (Ablauf des Verfahrens, Verfahrensdauer, beteiligte Personen etc.), um kontrollieren zu können, wie sich die Urteilsfindung gestaltet hat.

7 Im genauen Wortlaut: „Die zur Austragung des Spieles bestimmten Plätze sind nach den Fußballregeln zu zeichnen und nebst den erforderlichen Gerätschaften in gebrauchsfähigen Zustand zu setzen. Betreuern und Auswechselspielern wird bei den Spielen der Frauen und Herren ein speziell zu kennzeichnender Bereich zugewiesen, die Technische Zone. Diese erstreckt sich in einem Abstand von 10 Metern zur Mittellinie über 6 Meter und reicht in der Regel bis einen Meter an die Seitenlinie heran. In der Technischen Zone dürfen sich die auf dem Spielbericht benannten Auswechselspieler sowie weitere acht Mannschaftsverantwortliche aufhalten. Von der Technischen Zone aus dürfen taktische Anweisungen erteilt werden. Nur in Ausnahmefällen dürfen Trainer oder Betreuer die Technische Zone verlassen, zum Beispiel wenn der Schiedsrichter es gestattet, einen verletzten Spieler auf oder neben dem Feld zu behandeln. Der Schiedsrichter ist berechtigt, Personen aus der Technischen Zone hinter die Umzäunung auf die Zuschauerränge zu verweisen, wenn sich diese Personen mehrfach regelwidrig verhalten."

8 So titelte etwa die Neue Württembergische Zeitung am 12.07.2010 „Technische Zone erhitzt die Gemüter", die Schwäbische Zeitung am 13.07.2010 „Technische Zone sorgt für Gelächter“.

9 Für den Ablauf des Handschlags gibt es ein Protokoll (inklusive Skizze), das wie folgt lautet: „Die Heimmannschaft bleibt stehen. Die Gastmannschaft geht auf den Schiedsrichter und die Heimmannschaft zu. Im Vorbeigehen geben die Spieler dem Schiedsrichter und den Spielern der Heimmannschaft die Hand. Die Gastmannschaft geht auf seine [sic!] ursprüngliche Position zurück. Sobald der letzte Spieler der Gastmannschaft die Heimmannschaft passiert hat, führt der Spielführer der Heimmannschaft seine Mitspieler zum Handschlag am Schiedsrichter vorbei."

10 Beispielhaft für die Diskussion um die Ordnerpflicht sind etwa die Beiträge "Totaler Schwachsinn" vom 09.09.2010 und „Thema Sicherheit entzweit Verband und Vereine" vom 10.09.2010 in den Stuttgarter Nachrichten.

$11 \mathrm{Um}$ möglichst aussagekräftige Ergebnisse zu bekommen, wurde bei der Frageformulierung auf die Grundsätzlichkeit abgezielt, damit nicht einzelne Negativerlebnisse mit Ordnern das Gesamtbild verfälschen.

12 So wird in der Kriminologie auch der Begriff der „Risikogruppenprävention“ verwendet.

13 So werden beispielsweise für die Übernahme einer Spielleitung in der Bezirksliga in Württemberg derzeit $25 €$ Entschädigung gewährt. Im Vergleich hierzu erhält ein Schiedsrichter in der 1. Bundesliga ca. $3800 €$. 